\title{
Isometric and Closed-Range Composition Operators between Bloch-Type Spaces
}

\author{
Nina Zorboska \\ Department of Mathematics, University of Manitoba, Winnipeg, MB, Canada R3T 2N2 \\ Correspondence should be addressed to Nina Zorboska, zorbosk@cc.umanitoba.ca
}

Received 16 November 2010; Accepted 30 March 2011

Academic Editor: Asao Arai

Copyright (c) 2011 Nina Zorboska. This is an open access article distributed under the Creative Commons Attribution License, which permits unrestricted use, distribution, and reproduction in any medium, provided the original work is properly cited.

We present an overview of the known results describing the isometric and closed-range composition operators on different types of holomorphic function spaces. We add new results and give a complete characterization of the isometric univalently induced composition operators acting between Bloch-type spaces. We also add few results on the closed-range determination of composition operators on Bloch-type spaces and present the problems that are still open.

\section{Introduction}

A topic of interest in the paper is the description of isometric and, more generally, of closedrange composition operators on the Bloch-type spaces, in terms of the specific behaviour of the inducing function. The goal of the paper is to present an overview of the known results by emphasizing the intuitive idea and geometrical aspects of the corresponding conditions, to contribute to the classification with few new results and to list a number of open questions related to this topic.

One of the earliest results on isometric composition operators, acting on spaces of functions analytic on the open unit disk, is Nordgren's result [1] from 1968: if $\phi$ is inner, then $C_{\phi}$ is an isometry on $H^{2}$ if and only if $\phi(0)=0$. Martín and Vukotić have generalized recently in [2] that, indeed, $C_{\phi}$ is an isometry on $H^{p}$ for all $p \geq 1$ if and only if $\phi$ is inner and $\phi(0)=0$.

Since rotations induce isometric composition operators on most of the spaces, it is of particular interest to determine the function spaces on which these are the only kind of isometric composition operators. Such are, for example, all of the weighted Bergman spaces, as shown by Martín and Vukotić in [2]. They have also classified the isometric composition operators on the Dirichlet space and, under the univalence condition of the inducing function, 
on some of the other Besov spaces (see $[2,3]$ ). The isometric composition operators on the BMOA space have been determined by Laitila (see [4]).

As for the classification of the larger class of closed-range composition operators on spaces of functions analytic on the unit disk, the known results include some of the weighted Bergman spaces (see $[5,6]$ ), and some of the Bloch-type spaces, which we will state and refer to in the next few sections.

In most of the cases, the general rule is that a composition operator is either isometric or has a closed range, whenever the image of the unit disc $\mathbb{D}$ under the inducing function covers a significant (in some sense) part of $\mathbb{D}$. As we will see below, that stays true in the case of the Bloch-type spaces, with a specific description of what the "significant part" means in this context. Note that this represents a logical contrast to the description of the compact composition operators on all of these spaces, where the image of the unit disc under the inducing function must stay away significantly (again, in some sense) from the unit circle.

\section{Definitions, Few Basic Notions, and Overview of the Existing Results}

For a nonconstant analytic function $\phi$ that maps the unit disk $\mathbb{D}$ into itself, the composition operator $C_{\phi}$ on the Banach space $X \subseteq H(\mathbb{D})$ is defined by

$$
C_{\phi} f=f \circ \phi
$$

with $f$ in $X$, where $H(\mathbb{D})$ is the space of functions analytic on $\mathbb{D}$. We will say that $\phi$ is the inducing function for $C_{\phi}$.

Depending on the space $X$, one gets various conditions on the inducing function under which the corresponding composition operator satisfies a certain operator theoretic property such as, for example, being bounded, compact, invertible, normal, subnormal, isometric, closed range, Fredholm, and many others. For general results and references on composition operators acting on various spaces of analytic functions, see, for example, $[7,8]$.

For $\alpha>0$, the $\alpha$-Bloch spaces $B^{\alpha}$ (also referred to as Bloch-type spaces) are spaces of functions $f$ in $H(\mathbb{D})$ such that

$$
\||f|\|_{\mathbb{B}^{\alpha}}=\sup _{z \in \mathbb{D}}\left|f^{\prime}(z)\right|\left(1-|z|^{2}\right)^{\alpha}<\infty
$$

Each $B^{\alpha}$ is a Banach space with a norm given by

$$
\|f\|_{\mathbb{B}^{\alpha}}=|f(0)|+\||f|\|_{\mathbb{B}^{\alpha}} .
$$

The family of Bloch-type spaces includes the classical Bloch space $B=B^{1}$. The spaces $B^{\alpha}$ with $0<\alpha<1$ are the analytic Lipschitz spaces $\operatorname{Lip}_{1-\alpha}$. Thus, for $0<\alpha<1, B^{\alpha} \subset A(\mathbb{D}) \subset H^{\infty}$, where $A(\mathbb{D})$ is the disk algebra. In general, for $0<\alpha<\beta$, we have that $B^{\alpha} \subset B^{\beta}$ and so the $\alpha$-Bloch spaces $B^{\alpha}$ form an increasing, uniform family of function spaces. Note also that the Bloch space $B$ contains $H^{\infty}$ and is included in all of the Bergman spaces $L_{a}^{p}, p \geq 1$, while for large $\alpha$, such as $\alpha \geq 2, B^{\alpha}$ includes the Bergman space $L_{a}^{2}$. 
For further references and details on these general facts about the Bloch-type spaces stated above and more, see $[9,10]$.

The boundedness and compactness of composition operators acting between Blochtype spaces has been established in a series of papers [11-15]. We state the most general form of these results and use the following notation: for $\alpha>0, \beta>0$ and $\phi$ being an analytic self map of $\mathbb{D}$, let

$$
\tau_{\phi, \alpha, \beta}(z)=\frac{\left(1-|z|^{2}\right)^{\beta}\left|\phi^{\prime}(z)\right|}{\left(1-|\phi(z)|^{2}\right)^{\alpha}} .
$$

We write $\tau_{\phi, \alpha}$ whenever $\alpha=\beta$, and $\tau_{\phi}$ if $\alpha=\beta=1$.

Theorem $\mathbf{A}($ see $[12,15])$. For $\alpha, \beta>0$ and $\phi$ an analytic selfmap of $\mathbb{D}$, the composition operator $C_{\phi}$ is a bounded operator from $\boldsymbol{B}^{\alpha}$ into $\boldsymbol{B}^{\beta}$ if and only if

$$
\sup _{z \in \mathbb{D}} \tau_{\phi, \alpha, \beta}(z)<\infty
$$

and $C_{\phi}$ is a compact operator from $\boldsymbol{B}^{\alpha}$ into $\boldsymbol{B}^{\beta}$ if and only if

$$
\lim _{|\phi(z)| \rightarrow 1} \tau_{\phi, \alpha, \beta}(z)=0
$$

Here are a few simple consequences of Theorem A and of some basic complex analysis: recall the Schwarz-Pick lemma which states that for $\phi$ a self-map of the unit disk

$$
\frac{\left(1-|z|^{2}\right)\left|\phi^{\prime}(z)\right|}{1-|\phi(z)|^{2}} \leq 1 .
$$

Thus, when $\alpha=\beta=1, \tau_{\phi}(z) \leq 1$, and we get from Theorem A that every composition operator is bounded on $\boldsymbol{B}$. Moreover, the Schwarz-Pick lemma and Theorem A imply that if $\alpha \leq 1 \leq \beta$, then $C_{\phi}$ maps $B^{\alpha}$ boundedly into $\mathcal{B}^{\beta}$, since then $\tau_{\phi, \alpha, \beta}(z) \leq\left(1-|\phi(z)|^{2}\right)^{1-\alpha}$. If furthermore $\alpha<1 \leq \beta$, then $C_{\phi}$ from $B^{\alpha}$ into $B^{\beta}$ must also be compact. Note also that if $\|\phi\|_{\infty}<1$ and $C_{\phi}: \mathbb{B}^{\alpha} \rightarrow B^{\beta}$ is bounded, then $C_{\phi}$ is compact.

All of the spaces $B^{\alpha}$ include the identity function, and so a necessary condition for $C_{\phi}$ to be bounded from $B^{\alpha}$ into $B^{\beta}$ is that $\phi$ belongs to $B^{\beta}$. Thus, for $0<\beta<1$, every analytic self-map of $\mathbb{D}$ that is in $H^{\infty} \backslash B^{\beta}$ induces an unbounded composition operator from $\boldsymbol{B}^{\alpha}$ to $\boldsymbol{B}^{\beta}$.

In this paper, we are particularly interested in the closed-range composition operators and, even more specific, in the isometric composition operators. Recall that the operator $C_{\phi}$ : $\mathbb{B}^{\alpha} \rightarrow \mathbb{B}^{\beta}$ is isometric whenever

$$
\left\|C_{\phi} f\right\|_{\mathbb{B}^{\beta}}=\|f\|_{\mathbb{B}^{\alpha}}, \quad \forall f \in \mathbb{B}^{\alpha} .
$$

Since every nonconstant $\phi$ is an open map, the composition operator $C_{\phi}$ is always one to one. By a basic operator theory result, a one-to-one operator has a closed range if and only 
if it is bounded below. Thus, $C_{\phi}$ has a closed range if and only if it is bounded below, namely, if and only if there exists $M>0$ such that

$$
\left\|C_{\phi} f\right\|_{\mathcal{B}^{\beta}} \geq M\|f\|_{\mathcal{B}^{\alpha}}, \quad \forall f \in \mathbb{B}^{\alpha} .
$$

In particular, every isometric $C_{\phi}$ has a closed range.

On the other hand, recall that the only (closed) subspaces of the range of a compact operator are the finite dimensional ones. Thus, since a composition operator $C_{\phi}$ never has a finite rank (because $\phi$ is an open map), a compact $C_{\phi}$ can never have a closed range. Hence, a compact $C_{\phi}$ can never be an isometry.

The first few classification results on isometric composition operators acting on the Bloch-type spaces were done for the classical Bloch space in a series of papers [16-19]. We present the classification in the form that appears in [18].

Theorem B (see [18]). Let $\phi$ be an analytic self-map of $\mathbb{D}$. The composition operator $C_{\phi}$ is an isometry on the Bloch space $B$ if and only if $\phi(0)=0$ and either $\phi$ is a rotation, or for every $a$ in $\mathbb{D}$ there exists a sequence $\left\{z_{n}\right\}$ in $\mathbb{D}$ such that $\left|z_{n}\right| \rightarrow 1, \phi\left(z_{n}\right)=a$, and $\tau_{\phi}\left(z_{n}\right) \rightarrow 1$.

The result provides a large class of functions inducing isometric composition operators on the Bloch space. For example, if $\phi$ is an almost thin infinite Blaschke product fixing the origin, that is a Blaschke product with a sequence of zeroes $\left\{z_{n}\right\}$ that includes 0 and is such that $\limsup _{n \rightarrow \infty}\left|B^{\prime}\left(z_{n}\right)\right|\left(1-\left|z_{n}\right|^{2}\right)=1$, then $C_{\phi}$ is an isometry on $\boldsymbol{B}$. For more examples and the proof of the theorem, see [18].

For all of the other Bloch-type spaces, the result from [20] shows that the only isometric composition operators are the trivial ones.

Theorem C (see [20]). Let $0<\alpha, \alpha \neq 1$, and let $\phi$ be an analytic self-map of $\mathbb{D}$. Then the composition operator $C_{\phi}$ is an isometry on $B^{\alpha}$ if and only if $\phi$ is a rotation.

Remarks 2.1. As already mentioned in the introduction, one notices a general behavior of the functions inducing an isometric composition operator. From the two previous theorems, we can see that if $C_{\phi}$ is an isometry on $\mathbb{B}^{\alpha}$, then $\mathbb{D}=\phi(\mathbb{D})$, which further implies that $\partial \mathbb{D} \subseteq \overline{\phi(\mathbb{D})}$. Moreover, in the case $\alpha \neq 1, \phi$ must be a rotation.

This is similar to many other cases of isometric composition operators acting on spaces of analytic functions. The general requirement is that $\phi(\mathbb{D})$ covers a significant (in some sense) part of $\mathbb{D}$, or even further, that $\phi$ has to be a rotation. For example, if $C_{\phi}$ is an isometry either on the $H^{p}$ spaces or on the weighted Bergman spaces $A_{w}^{p}$ with $1 \leq p<\infty$, then $\phi$ has to be either an inner function or a rotation, respectively, as shown in [2]. If $C_{\phi}$ is an isometry on the Dirichlet space, then $\phi$ has to be an univalent full map, that is, one to one and such that the area measure of $\mathbb{D} \backslash \phi(\mathbb{D})$ is zero, (see [3]). The complete determination of isometric composition operators in all of this cases is given by adding the condition $\phi(0)=0$.

A specific isometric requirement in the case of the Bloch space is that when $\phi$ is not univalent, it has to be of infinite multiplicity and such that the function $\tau_{\phi}$ stays close to 1 over some of the preimages of each point in $\phi(\mathbb{D})$. When $\phi$ is univalent, $\phi$ has to be a rotation, and thus $\tau_{\phi}(z)=1$, for all $z \in \mathbb{D}$.

Since every isometric composition operator has a closed range, and since the closedrange composition operators are semi-Fredholm, that is, in some sense close to invertible, it 
is not too surprising that similar requirements on $\phi(\mathbb{D})$ and $\tau_{\phi, \alpha}$ play a role in determining the closed range composition operators on $B^{\alpha}$.

The following Theorem $\mathrm{D}$ below classifies the closed range composition operators on the Bloch spaces $B^{\alpha}, \alpha \geq 1$. The results are contained in a series of papers [21-23], and we present their combined version. We need a few more definitions before we can state the theorem.

We say that $G \subseteq \mathbb{D}$ is sampling for $\mathbb{B}^{\alpha}$ if $\exists S>0$ such that for all $f \in \mathbb{B}^{\alpha}$,

$$
\sup _{z \in G}\left|f^{\prime}(z)\right|\left(1-|z|^{2}\right)^{\alpha} \geq S\||f|\|_{B^{\alpha}}
$$

Let $\rho(z, w)=\left|\psi_{z}(w)\right|$ denote the pseudohyperbolic distance on $\mathbb{D}$, where $\psi_{z}$ is a disc automorphism of $\mathbb{D}$, that is,

$$
\psi_{z}(w)=\frac{z-w}{1-\bar{z} w}
$$

We say that $G \subseteq \mathbb{D}$ is an $r$ - net for $\mathbb{D}$ for some $r \in(0,1)$ if for all $z \in \mathbb{D}, \exists w \in G$ such that $\rho(z, w)<r$.

For $c>0$, let

$$
\Omega_{c, \alpha, \beta}=\left\{z \in \mathbb{D}: \tau_{\phi, \alpha, \beta}(z) \geq c\right\}
$$

and let $G_{c, \alpha, \beta}=\phi\left(\Omega_{c, \alpha, \beta}\right)$. If $\alpha=\beta$, we use the notation $\Omega_{c, \alpha}$ and $G_{c, \alpha}$, and if further $\alpha=\beta=1$, we write $\Omega_{c}$ and $G_{c}$.

Theorem $\mathbf{D}$ (see $[21,23,24])$. Let $\phi$ be a self-map of $\mathbb{D}$ and let $\alpha \geq 1$. Then, the following are equivalent.

(i) $C_{\phi}$ has a closed range on $B^{\alpha}$.

(ii) There exists $c>0$ such that the set $G_{c, \alpha}$ is sampling for $B^{\alpha}$.

(iii) There exist $c, r>0$ with $r<1$ such that $G_{c, \alpha}$ is an $r$-net for $\mathbb{D}$.

Moreover, if $\alpha=1$, then (i) to (iii) are also equivalent to the following.

(iv) There exists $k>0$ such that $\left\|C_{\phi} \psi_{a}\right\|_{\mathcal{B}} \geq k\left\|\psi_{a}\right\|_{\mathcal{B}}$, for all $a \in \mathbb{D}$.

Note, for example, that when $\alpha=1$ and $\phi$ is a rotation, $\tau_{\phi}(z)=1$, for all $z \in \mathbb{D}$, and so $G_{1}=\mathbb{D}$. Thus, $G_{1}$ is trivially sampling for $B$ and an $r$-net for $\mathbb{D}$ for any $r>0$. Therefore, (ii) and (iii) are true. Part (iv) of the theorem also holds true if one takes $k=1$.

If $\phi$ is not a rotation and $C_{\phi}$ is an isometry on $B$, then whenever $c=1-\varepsilon$ with $0<\varepsilon<1$, we have from Theorem $B$ that $G_{1-\varepsilon}=\mathbb{D}$. Hence, as before, $G_{1-\varepsilon}$ is trivially sampling for $B$ and an $r$-net for $\mathbb{D}$, for any $r>0$. Again, part (iv) of the theorem is true with $k=1$. Thus, we get a geometric description of closed-range (or isometric) composition operators on these Bloch spaces as composition operators for which the inducing function $\phi$ has a $\tau_{\phi, \alpha}$ function that stays away from zero (or is close enough to one) over a set with large enough image. 
The geometrical aspects of the restrictions on the $\tau_{\phi}$ function are particularly interesting in the case when $\phi$ is univalent, since then, as a consequence of the Köebe onequarter theorem, we have that

$$
\tau_{\phi}(z) \approx \frac{\operatorname{dist}(\phi(z), \partial \phi(\mathbb{D}))}{1-|\phi(z)|}
$$

For the isometry case, this implies that $\phi$ has to be a rotation, and for the closedrange case, it gives a deeper insight on the boundary behavior of $\phi$, providing a number of interesting examples and counterexamples.

For example, one gets that if $\phi$ is the Riemann mapping from $\mathbb{D}$ onto the slit disk $\mathbb{D} \backslash[0,1]$, then $C_{\phi}$ has a closed range on $\mathcal{B}$. Or if $\phi$ is the Riemann mapping onto the simply connected region in $\mathbb{D}$ created by taking away an infinite countable number of slits and pseudo-hyperbolic disks connected to the slits, one can get either a closed-range or a nonclosed-range composition operator $C_{\phi}$ by controlling the size and the placement of the pseudohyperbolic disks. For more details on these examples, see [23].

It is not too hard to see that if $C_{\phi}$ has a closed range on $B^{\alpha}$ for some $\alpha>1$, then $C_{\phi}$ has a closed range on $B^{\alpha}$ for all $\alpha \geq 1$. On the other hand, the slit disk example from above provides an example of a univalent map $\phi$ such that $C_{\phi}$ has a closed range on $B^{\alpha}$ only for $\alpha=1$ (see $[21,23])$.

\section{Further Results on Closed-Range and Isometric Composition Operators on the Bloch-Type Spaces}

In this section, we present our results on the classification of the isometric and closed-range composition operators $C_{\phi}: B^{\alpha} \rightarrow B^{\beta}$. As Theorem A from the previous section states, not every such composition operator is bounded. Thus, the boundedness condition on $\tau_{\phi, \alpha, \beta}$ plays a natural role in the characterization of the isometric and closed-range composition operators from $B^{\alpha}$ into $B^{\beta}$.

In general, depending on the choices of $\alpha$ and $\beta$, a composition operator induced by a specific, fixed function can behave very differently. We illustrate this with the following example, in which the inducing function is one of the nicest univalent selfmaps of the unit disk, namely, a rotation.

Example 3.1. Let $\phi(z)=\lambda z$, with $|\lambda|=1$. Then $C_{\phi}: \mathbb{B}^{\alpha} \rightarrow \mathbb{B}^{\beta}$ is

(a) an isometry, whenever $\alpha=\beta$,

(b) a compact operator if $\alpha<\beta$,

(c) an unbounded operator if $\alpha>\beta$.

Note that (a) holds since $\left|\phi^{\prime}(z)\right|=|\lambda|=1$, and we have that

$$
\left\|C_{\phi} f\right\|_{\mathbb{B}^{\alpha}}=\|f \circ \phi\|_{\mathcal{B}^{\alpha}}=|f(0)|+\sup _{z \in \mathbb{D}}\left|f^{\prime}(\lambda z)\right|\left(1-|\lambda z|^{2}\right)^{\alpha}=\|f\|_{B^{\alpha}} .
$$


The other two cases follow easily from Theorem A, since

$$
\tau_{\phi, \alpha, \beta}=\frac{\left(1-|z|^{2}\right)^{\beta}\left|\phi^{\prime}(z)\right|}{\left(1-|\phi(z)|^{2}\right)^{\alpha}}=\left(1-|z|^{2}\right)^{\beta-\alpha} .
$$

As for many other spaces, a condition that $\phi$ must satisfy whenever $C_{\phi}$ is an isometry from $B^{\alpha}$ into $B^{\beta}$, regardless of the choices of $\alpha$ and $\beta$, is that $\phi(0)=0$.

Proposition 3.2. If $C_{\phi}$ is an isometry from $\mathbb{B}^{\alpha}$ into $\boldsymbol{B}^{\beta}$, then $\phi(0)=0$ and

$$
\sup _{z \in \mathbb{D}} \tau_{\phi, \alpha, \beta}(z)=1
$$

Proof. Note first that the identity function $e(z)=z$ belongs to each of the Bloch-type spaces and has norm one. Thus, since $C_{\phi}$ is an isometry, it must be that $\|\phi\|_{\mathcal{B}^{\beta}}=\left\|C_{\phi} e\right\|_{\mathcal{B}^{\beta}}=\|e\|_{\mathcal{B}^{\alpha}}=1$.

Let $\phi(0)=a$. Using the function $f_{a}(z)=1-\bar{a} z$, we see that

$$
\begin{aligned}
\left\|C_{\phi} f_{a}\right\|_{\mathcal{B}^{\beta}} & =\left\|f_{a} \circ \phi\right\|_{\mathcal{B}^{\beta}}=\left|f_{a}(a)\right|+\sup _{z \in \mathbb{D}}\left|f_{a}^{\prime}(\phi(z))\right|\left|\phi^{\prime}(z)\right|\left(1-|z|^{2}\right)^{\beta} \\
& =1-|a|^{2}+|a| \sup _{z \in \mathbb{D}}\left|\phi^{\prime}(z)\right|\left(1-|z|^{2}\right)^{\beta}=1-|a|^{2}+|a|(1-|a|) \\
& =1+|a|-2|a|^{2} .
\end{aligned}
$$

But since $C_{\phi}$ is an isometry and $\left\|f_{a}\right\|_{B^{\alpha}}=1+|a| \sup _{z \in \mathbb{D}}\left(1-|z|^{2}\right)^{\alpha}=1+|a|$, it must be that $|a|^{2}=0$. Thus $\phi(0)=a=0$.

To show that $\sup _{z \in \mathbb{D}} \tau_{\phi, \alpha, \beta}(z)=1$, we use another type of test functions. For $a \in \mathbb{D}$, let $\varphi_{a}(z)$ be the function such that $\varphi_{a}(0)=0$ and $\varphi_{a}^{\prime}(z)=\left(\psi_{a}^{\prime}(z)\right)^{\alpha}$, where $\psi_{a}$ is the disc automorphism of $\mathbb{D}$ defined by

$$
\psi_{a}(z)=\frac{a-z}{1-\bar{a} z}
$$

Using that $\left(1-|z|^{2}\right)\left|\psi_{w}^{\prime}(z)\right|=1-\left|\psi_{w}(z)\right|^{2}$, we get

$$
\begin{aligned}
\left\|\varphi_{a}\right\|_{\mathbb{B}^{\alpha}} & =\sup _{z \in \mathbb{D}}\left|\psi_{a}^{\prime}(z)\right|^{\alpha}\left(1-|z|^{2}\right)^{\alpha} \\
& =\sup _{z \in \mathbb{D}}\left(1-\left|\psi_{a}(z)\right|^{2}\right)^{\alpha}=1 .
\end{aligned}
$$


Hence, since $C_{\phi}$ is an isometry from $\mathbb{B}^{\alpha}$ into $\mathbb{B}^{\beta}$, we have that for any $z_{0}$ in $\mathbb{D}$

$$
\begin{aligned}
1 & =\sup _{a \in \mathbb{D}}\left\|C_{\phi} \varphi_{a}\right\|_{\mathcal{B}^{\beta}}=\operatorname{supsup}_{a \in \mathbb{D}} \tau_{z \in \mathbb{D}}(z, \beta)\left(1-\left|\psi_{a}(\phi(z))\right|^{2}\right)^{\alpha} \\
& \geq \sup _{a \in \mathbb{D}} \tau_{\phi, \alpha, \beta}\left(z_{0}\right)\left(1-\left|\psi_{a}\left(\phi\left(z_{0}\right)\right)\right|^{2}\right)^{\alpha} \\
& \geq \tau_{\phi, \alpha, \beta}\left(z_{0}\right),
\end{aligned}
$$

where the last inequality follows by choosing $a=\phi\left(z_{0}\right)$, and the fact that $\psi_{a}(a)=0$. Thus $\tau_{\phi, \alpha, \beta}(z) \leq 1$, for all $z \in \mathbb{D}$. On the other hand, for any $a \in \mathbb{D}$

$$
1=\left\|C_{\phi} \varphi_{a}\right\|_{\mathcal{B}^{\beta}}=\sup _{z \in \mathbb{D}} \tau_{\phi, \alpha, \beta}(z)\left(1-\left|\psi_{a}(z)\right|^{2}\right)^{\alpha} \leq \sup _{z \in \mathbb{D}} \tau_{\phi, \alpha, \beta}(z)
$$

and thus, $\sup _{z \in \mathbb{D}} \tau_{\phi, \alpha, \beta}(z)=1$.

Note that in the more general case when $C_{\phi}: \mathbb{B}^{\alpha} \rightarrow \boldsymbol{B}^{\beta}$ has a closed range, $\phi(0)$ does not necessarily have to be zero. Still, without loss of generality, we will consider only the case $\phi(0)=0$. This is possible since the disk automorphisms induce invertible (and thus closed range) composition operators on every Bloch-type space. Namely, if $\phi(0)=a$, we have that $\phi_{a}(z)=\psi_{a} \circ \phi(z)$ is such that $\phi_{a}(0)=0$ and $C_{\phi_{a}}=C_{\phi} C_{\psi_{a}}$. Moreover,

$$
\tau_{\psi_{a}, \alpha}(z)=\left|\psi_{a}^{\prime}(z)\right|^{1-\alpha} \leq\left(\frac{1+|a|}{1-|a|}\right)^{|1-\alpha|}
$$

and by Theorem A we have that $C_{\psi_{a}}: B^{\alpha} \rightarrow B^{\alpha}$ is always a bounded (and an invertible) operator. Thus $C_{\phi}: \mathbb{B}^{\alpha} \rightarrow \mathcal{B}^{\beta}$ has a closed range if and only if $C_{\phi_{a}}: \mathbb{B}^{\alpha} \rightarrow \mathcal{B}^{\beta}$ has a closed range.

Theorem 3.3. Let $\phi$ be a selfmap of $\mathbb{D}$, let $\alpha, \beta>0$, and let $C_{\phi}: \mathbb{B}^{\alpha} \rightarrow \mathbb{B}^{\beta}$ be bounded. Then

(i) $C_{\phi}: B^{\alpha} \rightarrow B^{\beta}$ has a closed range if and only if there exists $c>0$ such that the set $G_{c, \alpha, \beta}$ is sampling for $B^{\alpha}$;

(ii) if $C_{\phi}: \mathbb{B}^{\alpha} \rightarrow \mathbb{B}^{\beta}$ has a closed range, then there exist $c, r>0$ with $r<1$, such that $G_{c, \alpha, \beta}$ is an $r$-net for $\mathbb{D}$;

(iii) if there exist $c, r>0$ with $r<1$ such that $G_{c, \alpha, \beta}$ contains an open annulus centered at the origin and with outer radius 1 , then $C_{\phi}$ has a closed range.

Proof. The proof of (i) is similar to the proof of Theorem 1 from [23]. Since $C_{\phi}: \mathbb{B}^{\alpha} \rightarrow \mathbb{B}^{\beta}$ is bounded, by Theorem $\mathrm{A}$, we have that $\exists K>0$ such that $\tau_{\phi, \alpha, \beta}(z) \leq K$. Thus, if $C_{\phi}$ is bounded below by $M$, and if $c=M / 2$, we will show that set $G_{c, \alpha, \beta}$ is sampling for $\mathbb{B}^{\alpha}$ with a sampling constant $S=M / K$. We have that for any $f \in \mathbb{B}^{\alpha}$,

$$
\left\|C_{\phi} f\right\|_{\mathcal{B}^{\beta}}=\sup _{z \in \mathbb{D}} \tau_{\phi, \alpha, \beta}(z)\left(1-|\phi(z)|^{2}\right)^{\alpha}\left|f^{\prime}(\phi(z))\right| \geq M\|f\|_{\mathcal{B}^{\alpha}}
$$


and since

$$
\sup _{z \notin \Omega_{c, \alpha, \beta}} \tau_{\phi, \alpha, \beta}(z)\left(1-|\phi(z)|^{2}\right)^{\alpha}\left|f^{\prime}(\phi(z))\right|<c\|f\|_{\mathbb{B}^{\alpha}}=\frac{M}{2}\|f\|_{\mathcal{B}^{\alpha} \prime}
$$

it must be that

$$
\begin{aligned}
M\|f\|_{\mathcal{B}^{\alpha}} & \leq \sup _{z \in \mathbb{D}} \tau_{\phi, \alpha, \beta}(z)\left(1-|\phi(z)|^{2}\right)^{\alpha}\left|f^{\prime}(\phi(z))\right| \\
& =\sup _{z \in \Omega_{c, \alpha, \beta}} \tau_{\phi, \alpha, \beta}(z)\left(1-|\phi(z)|^{2}\right)^{\alpha}\left|f^{\prime}(\phi(z))\right| \\
& \leq K \sup _{w \in G_{c, \alpha, \beta}}\left(1-|w|^{2}\right)^{\alpha}\left|f^{\prime}(w)\right| .
\end{aligned}
$$

Thus $\sup _{w \in G_{c, \alpha, \beta}}\left(1-|w|^{2}\right)^{\alpha}\left|f^{\prime}(w)\right| \geq M / K\|f\|_{\mathcal{B}^{\alpha}}$, that is, $G_{c, \alpha, \beta}$ is sampling for $\mathbb{B}^{\alpha}$ with sampling constant $S=M / K$. The other direction of the proof is fairly similar, and we leave it to the reader.

(ii) Let $w \in \mathbb{D}$ and let $\varphi_{w}$ be as in the proof of Proposition 3.2, that is, the function defined by $\varphi_{w}(0)=0$ and $\varphi_{w}^{\prime}=\left(\psi_{w}^{\prime}\right)^{\alpha}$. As shown before, $\left\|\varphi_{w}\right\|_{\mathcal{B}^{\alpha}}=1$, and

$$
\left\|\varphi_{w} \circ \phi\right\|_{\mathcal{B}^{\beta}}=\sup _{z \in \mathbb{D}} \tau_{\phi, \alpha, \beta}(z)\left(1-\left|\psi_{w}(\phi(z))\right|^{2}\right)^{\alpha}
$$

Furthermore, assuming that $C_{\phi}$ is bounded and has a closed range, there exist $M, K>0$ such that $\sup _{z \in \mathbb{D}} \tau_{\phi, \alpha, \beta}(z)=K$ and

$$
K \geq \sup _{z \in \mathbb{D}} \tau_{\phi, \alpha, \beta}(z)\left(1-\left|\psi_{w}(\phi(z))\right|^{2}\right)^{\alpha} \geq M
$$

But since $1-\left|\psi_{w}(\phi(z))\right|^{2} \leq 1$, there exists $z_{w} \in \mathbb{D}$ such that

$$
\tau_{\phi, \alpha, \beta}\left(z_{w}\right) \geq \frac{M}{2}, \quad\left(1-\left|\psi_{w}\left(\phi\left(z_{w}\right)\right)\right|^{2}\right)^{\alpha} \geq \frac{M}{2 K}
$$

Thus, for $c=M / 2$ and $r=\sqrt{1-(M / 2 K)^{1 / \alpha}}$, we have that for all $w \in \mathbb{D}$; there exists $z_{w} \in$ $\Omega_{c, \alpha, \beta}$ such that $\rho\left(w, \phi\left(z_{w}\right)\right)<r$, and so $G_{c, \alpha, \beta}$ is an $r$-net for $\mathbb{D}$.

(iii) Let $C_{\phi}: \mathbb{B}^{\alpha} \rightarrow B^{\beta}$ be bounded and assume that $G_{c, \alpha, \beta}$ contains the annulus $A=\{z$ : $\left.r_{0}<|z|<1\right\}$. Suppose that $C_{\phi}$ does not have a closed range, that is, there exists a sequence of functions $\left\{f_{n}\right\}$ with $\left\|f_{n}\right\|_{\mathbb{B}^{\alpha}}=1$ and such that $\left\|C_{\phi} f_{n}\right\|_{\mathcal{B}^{\beta}} \rightarrow 0$. Since $\sup _{z \in \mathbb{D}}\left(1-|z|^{2}\right)^{\alpha}\left|f_{n}^{\prime}(z)\right|=1$, there exists a sequence $\left\{a_{n}\right\}$ in $\mathbb{D}$ such that for all $n$,

$$
\left(1-\left|a_{n}\right|^{2}\right)^{\alpha}\left|f_{n}^{\prime}\left(a_{n}\right)\right| \geq \frac{1}{2}
$$


For any $\varepsilon>0$, let $N_{\varepsilon}$ be such that for all $n>N_{\varepsilon}$, and we have $\left\|C_{\phi} f_{n}\right\|_{\mathcal{B}^{\beta}}<c \varepsilon$. Then

$$
\begin{aligned}
\sup _{w \in G_{c, \alpha, \beta}} & \left(1-|w|^{2}\right)^{\alpha}\left|f_{n}^{\prime}(w)\right| \\
\leq & \frac{1}{c} \sup _{z \in \Omega_{c, \alpha, \beta}} \tau_{\phi, \alpha, \beta}(z)\left(1-|\phi(z)|^{2}\right)^{\alpha}\left|f_{n}^{\prime}(\phi(z))\right| \\
\quad \leq & \frac{1}{c} \sup _{z \in \mathbb{D}}\left(1-|z|^{2}\right)^{\beta}\left|f_{n}^{\prime}(\phi(z))\right|\left|\phi^{\prime}(z)\right|=\frac{1}{c}\left\|C_{\phi} f_{n}\right\|_{\mathcal{B}^{\beta}}<\frac{c \mathcal{E}}{c}=\varepsilon .
\end{aligned}
$$

Considering further $\varepsilon<1 / 2$, we get that each $a_{n}$ with $n>N_{\varepsilon}$ belongs to the complement of $G_{c, \alpha, \beta}$. Thus $\left|a_{n}\right| \leq r_{0}<1$ and $a_{n} \rightarrow a$ with $|a| \leq r_{0}$.

On the other hand, since $\left\|f_{n}\right\|_{\mathcal{B}^{\alpha}}=1$, a normal families argument implies that there exists a subsequence $\left\{f_{n_{m}}\right\}$ that converges uniformly on compact subsets of $\mathbb{D}$ to some function $f \in \mathbb{B}^{\alpha}$. But then $\left\{f_{n_{m}}^{\prime}\right\}$ converges to $f^{\prime}$ uniformly on compact subsets of $\mathbb{D}$, and since $\sup _{w \in G_{c, \alpha, \beta}}\left(1-|w|^{2}\right)^{\alpha}\left|f_{n}^{\prime}(w)\right| \rightarrow 0$ as $n \rightarrow \infty$ and $G_{c, \alpha, \beta}$ contains an infinite compact subset of $\mathbb{D}$, we get that $f^{\prime} \equiv 0$. This contradicts the fact that $\left(1-|a|^{2}\right)^{\alpha}\left|f^{\prime}(a)\right| \geq 1 / 2$ and so $C_{\phi}$ must be bounded below. Hence, $C_{\phi}$ has a closed range.

Example 3.4. Let $\phi(z)=z^{2}$, and let $C_{\phi}: \mathbb{B}^{\alpha} \rightarrow \mathbb{B}^{\beta}$. Then

$$
\tau_{\phi, \alpha, \beta}(z)=\frac{2|z|\left(1-|z|^{2}\right)^{\beta}}{\left(1-|z|^{4}\right)^{\alpha}}=\left(1-|z|^{2}\right)^{\beta-\alpha} \frac{2|z|}{\left(1+|z|^{2}\right)^{\alpha}} .
$$

For $z \in \mathbb{D}$ with $|z|>1 / 2$, we have that $\left(1 / 2^{\alpha}\right)\left(1-|z|^{2}\right)^{\beta-\alpha}<\tau_{\phi, \alpha, \beta}(z) \leq 2\left(1-|z|^{2}\right)^{\beta-\alpha}$. Thus

(i) if $\alpha<\beta$, we have that $\tau_{\phi, \alpha, \beta}(z) \rightarrow 0$ as $|z| \rightarrow 1$, and so $C_{\phi}$ is compact;

(ii) if $\alpha>\beta$, we have that $\tau_{\phi, \alpha, \beta}(z) \rightarrow \infty$ as $|z| \rightarrow 1$, and so $C_{\phi}$ is not bounded;

(iii) if $\alpha=\beta$, we have that $\left(1 / 2^{\alpha}\right)<\tau_{\phi, \alpha}(z) \leq 2$, that is, $A=\{z:(1 / 2)<|z|<1\} \subset G_{1 / 2^{\alpha}, \alpha}$ and so $C_{\phi}$ is bounded below and has a closed range. Recall that $C_{\phi}$ cannot be an isometry on any of the $B^{\alpha}$ spaces.

Note that the same conclusions as in Example 3.4 hold if we choose $\phi(z)=z^{k}, k \in \mathbb{N}$, or even further, if we choose other particularly nice functions, such as, for example, finite Blaschke products. The sufficient condition, as we will see later, is the boundedness of the derivative over points that are mapped close to the unit circle.

As for the boundedness of the composition operator from $\mathcal{B}^{\alpha}$ into $\mathcal{B}^{\beta}$ when $\alpha>\beta$ in general, we mention the following useful condition which might be known, but we could not find it in the literature.

Proposition 3.5. Let $0<\beta<\alpha$, and let $\phi$ be an analytic selfmap of $\mathbb{D}$. If the composition operator $C_{\phi}$ from $\mathbb{B}^{\alpha}$ into $\mathbb{B}^{\beta}$ is bounded, then $\phi$ has no angular derivative on $\partial \mathbb{D}$, and if $E=\{\zeta \in \partial \mathbb{D}:|\phi(\zeta)|=1\}$, then the (linear) measure of E must be zero. 
Proof. Recall that if $\phi$ has an angular derivative at a point $\zeta \in \partial \mathbb{D}$, then $|\phi(\zeta)|=1$. Thus, if the set $E$ is empty, we are done. If not, recall further that if $\phi$ has an angular derivative $\phi^{\prime}(\zeta)$ at $\zeta$, then for any sequence $\left\{z_{n}\right\}$ in $\mathbb{D}$ that converges nontangentially to $\zeta$, we have that

$$
\lim _{n \rightarrow \infty} \frac{1-\left|\phi\left(z_{n}\right)\right|}{1-\left|z_{n}\right|}=\left|\phi^{\prime}(\zeta)\right|=\lim _{n \rightarrow \infty}\left|\phi^{\prime}\left(z_{n}\right)\right|
$$

Also, if $\phi(0)=0$, it must be that $\left|\phi^{\prime}(\zeta)\right| \geq 1$. For more details on angular derivatives, see, for example, [8].

Without loss of generality, assume that $\phi(0)=0$ and suppose that $\phi$ has an angular derivative at some $\zeta \in \partial \mathbb{D}$. Hence, whenever $\left\{z_{n}\right\}$ in $\mathbb{D}$ converges nontangentially to $\zeta$, we have that for large enough $n$,

$$
\tau_{\phi, \alpha, \beta}\left(z_{n}\right)=\frac{\left(1-\left|z_{n}\right|^{2}\right)^{\beta}\left|\phi^{\prime}\left(z_{n}\right)\right|}{\left(1-\left|\phi\left(z_{n}\right)\right|^{2}\right)^{\alpha}} \geq \frac{1}{2}\left(\frac{\left(1-\left|z_{n}\right|^{2}\right)}{\left(1-\left|\phi\left(z_{n}\right)\right|^{2}\right)}\right)^{\beta} \frac{1}{\left(1-\left|\phi\left(z_{n}\right)\right|^{2}\right)^{\alpha-\beta}} \rightarrow \infty
$$

as $n \rightarrow \infty$, since $\left(1-\left|z_{n}\right|^{2}\right) /\left(1-\left|\phi\left(z_{n}\right)\right|^{2}\right) \rightarrow\left|\phi^{\prime}(\zeta)\right|^{-\beta}$ and $\left|\phi\left(z_{n}\right)\right| \rightarrow 1$. By Theorem A, $C_{\phi}$ cannot be bounded from $B^{\alpha}$ into $B^{\beta}$.

Furthermore, by a result from [14, Corollary, page 71], if the set $E$ has a positive measure in $\partial \mathbb{D}$, then $\phi$ must have an angular derivative at some point of $\partial \mathbb{D}$, and so $C_{\phi}$ can not be bounded from $\AA^{\alpha}$ into $\AA^{\beta}$.

Recall that Theorems B, C, and D consider the closed-range and isometry classifications of composition operators acting between the same space $\mathbb{B}^{\alpha}$. The following theorem looks at the determination of closed-range and isometric composition operators acting between different Bloch-type spaces, namely, the case when $\alpha \neq \beta$.

Theorem 3.6. Let $\alpha, \beta>0, \alpha \neq \beta$, and let $C_{\phi}$ from $\boldsymbol{B}^{\alpha}$ into $\boldsymbol{B}^{\beta}$ be bounded. Then

(i) $C_{\phi}$ can not have a closed range, except possibly when $0<\alpha<\beta<1$, or $1 \leq \beta<\alpha$;

(ii) $C_{\phi}$ can not be an isometry, except possibly when $0<\alpha<\beta<1$.

Proof. (i)

Case 1. Let $\alpha>\beta$. We will show that if $\alpha>\beta$ and $\beta<1$, then $C_{\phi}$ bounded implies that $\|\phi\|_{\infty}<1$ and so, by Theorem $\mathrm{A}, C_{\phi}$ is compact. Thus $C_{\phi}$ can not have a closed range.

This follows from the more general fact that if $C_{\phi}: B^{\alpha} \rightarrow B^{\beta}$ is bounded, then for $0<\varepsilon<\alpha$ we have that $C_{\phi}: \mathbb{B}^{\alpha-\varepsilon} \rightarrow \mathbb{B}^{\beta}$ must be compact, since

$$
\frac{\left(1-|z|^{2}\right)^{\beta}\left|\phi^{\prime}(z)\right|}{\left(1-|\phi(z)|^{2}\right)^{\alpha-\varepsilon}} \leq\left(1-|\phi(z)|^{2}\right)^{\varepsilon} \sup _{z \in \mathbb{D}} \frac{\left(1-|z|^{2}\right)^{\beta}\left|\phi^{\prime}(z)\right|}{\left(1-|\phi(z)|^{2}\right)^{\alpha}}
$$

which converges to 0 , as $|\phi(z)| \rightarrow 1$. 
So, since $0<\varepsilon=\alpha-\beta<\alpha$, we have that if $C_{\phi}: \mathbb{B}^{\alpha} \rightarrow B^{\beta}$ is bounded, then $C_{\phi}: \mathbb{B}^{\beta} \rightarrow \mathbb{B}^{\beta}$ is compact, and since $\beta<1$, it must be that $\|\phi\|_{\infty}<1$ as shown, for example, in [14].

Case 2. Let $\alpha<\beta$.

(a) If $\alpha \geq 1$, then each $C_{\phi}$ is compact and thus can not have a closed range. This follows from the fact that

$$
\tau_{\phi, \alpha, \beta}(z)=\frac{\left(1-|z|^{2}\right)^{\beta}\left|\phi^{\prime}(z)\right|}{\left(1-|\phi(z)|^{2}\right)^{\alpha}} \leq \frac{\left(1-|z|^{2}\right)\left|\phi^{\prime}(z)\right|}{1-|\phi(z)|^{2}}\left(1-|z|^{2}\right)^{\beta-\alpha} \leq\left(1-|z|^{2}\right)^{\beta-\alpha} .
$$

The first inequality is true since $\phi(0)=0$ and so

$$
\frac{1}{\left(1-|\phi(z)|^{2}\right)^{\alpha-1}} \leq \frac{1}{\left(1-|z|^{2}\right)^{\alpha-1}}
$$

while in the second inequality one uses the Schwarz-Pick lemma. Again, since $\phi(0)=0$, we have that $1-|z|^{2} \leq 1-|\phi(z)|^{2}$, and by Theorem A, $C_{\phi}$ must be compact.

(b) When $\alpha<1 \leq \beta$, then again, each $C_{\phi}$ is compact and so, $C_{\phi}$ can not have a closed range. This follows similarly from the Schwarz-Pick lemma and Theorem A:

$$
\begin{aligned}
\frac{\left(1-|z|^{2}\right)^{\beta}\left|\phi^{\prime}(z)\right|}{\left(1-|\phi(z)|^{2}\right)^{\alpha}} & =\left(\frac{\left(1-|z|^{2}\right)\left|\phi^{\prime}(z)\right|}{1-|\phi(z)|^{2}}\right)^{\alpha}\left(1-|z|^{2}\right)^{1-\alpha}\left|\phi^{\prime}(z)\right|^{1-\alpha}\left(1-|z|^{2}\right)^{\beta-1} \\
& \leq\left(1-|\phi(z)|^{2}\right)^{1-\alpha}\left(1-|z|^{2}\right)^{\beta-1} \rightarrow 0
\end{aligned}
$$

as $|\phi(z)| \rightarrow 1$.

(ii) Since every isometry has a closed range, by part (i), the leftover case to consider when determining the isometric composition operators $C_{\phi}$ from $B^{\alpha}$ into $B^{\beta}$ is when $1 \leq \beta<\alpha$. We will show that in this case also, the composition operator can not be an isometry.

(a) Let $1<\beta<\alpha$. Then, if $C_{\phi}: \mathbb{B}^{\alpha} \rightarrow \mathcal{B}^{\beta}$ is an isometry, we have that $\|\phi\|_{\mathcal{B}^{\beta}}=1$. Thus

$$
\sup _{z \in \mathbb{D}}\left(1-|z|^{2}\right)^{\beta-1}\left(1-|z|^{2}\right)\left|\phi^{\prime}(z)\right|=1
$$

where $\left(1-|z|^{2}\right)^{\beta-1} \leq 1$ and $\left(1-|z|^{2}\right)\left|\phi^{\prime}(z)\right| \leq 1-|\phi(z)|^{2} \leq 1$. Therefore, the supremum is 1 only when both parts of the product converge or are equal to 1 . This implies that the supremum is attained at $z=0$ and that $\left|\phi^{\prime}(0)\right|=1$. But then, by Schwarz's lemma, $\phi$ must be a rotation, and rotations induce an unbounded composition operators whenever $\beta<\alpha$. Hence, a composition operator from $\boldsymbol{B}^{\alpha} \rightarrow \boldsymbol{B}^{\beta}$ is never an isometry in the case $1<\beta<\alpha$. 
(b) If $1=\beta<\alpha$ and $C_{\phi}: \mathbb{B}^{\alpha} \rightarrow \mathcal{B}$ is an isometry, then, as before, $\|\phi\|_{\mathcal{B}}=1$. In the proof of Theorem B in [18], it is shown that this is enough to imply that either $\phi$ is a rotation (but then $C_{\phi}$ is not bounded) or that $\phi$ is such that for every $a$ in $\mathbb{D}$ there exists $\left\{z_{n}\right\}$ with $z_{n} \rightarrow 1, \phi\left(z_{n}\right)=a$, and $\tau_{\phi}\left(z_{n}\right) \rightarrow 1$.

Let $\left\{a_{k}\right\}$ be a sequence of points in $\mathbb{D}$ such that $\left|a_{k}\right| \rightarrow 1$, and let $\left\{z_{k}\right\}$ be such that $\phi\left(z_{k}\right)=a_{k}$ and $\tau_{\phi}\left(z_{k}\right) \geq 1 / 2$. Then, as $a_{k} \rightarrow 1$,

$$
\tau_{\phi, \alpha, 1}\left(z_{k}\right)=\frac{\left(1-\left|z_{k}\right|^{2}\right)\left|\phi^{\prime}\left(z_{k}\right)\right|}{\left(1-\left|\phi\left(z_{k}\right)\right|^{2}\right)^{\alpha}}=\frac{\tau_{\phi}\left(z_{k}\right)}{\left(1-\left|a_{k}\right|^{2}\right)^{\alpha-1}} \geq \frac{1}{2\left(1-\left|a_{k}\right|^{2}\right)^{\alpha-1}} \rightarrow \infty,
$$

and so $C_{\phi}: \mathbb{B}^{\alpha} \rightarrow B$ is not bounded.

Note that in the proof of the previous theorem, we have shown that in general, whenever $\phi(0)=0,\|\phi\|_{\mathcal{B}^{\beta}}=1$, and $1 \leq \beta<\alpha$, then $C_{\phi}: \mathbb{B}^{\alpha} \rightarrow \mathcal{B}^{\beta}$ is not bounded.

We state one more result on the isometric and closed-range composition operators on Bloch-type spaces, which takes care of the classification in the case when the inducing function $\phi$ satisfies extra conditions. For example, we show that the only univalently induced isometric composition operators are the trivial ones, that is, the ones induced by rotations. This is similar to a result from [25], where we have shown that a univalently induced composition operator on $\mathbb{B}^{\alpha}, \alpha \neq 1$, has a closed range if and only if $\phi$ is a disk automorphism.

Theorem 3.7. Let $\alpha, \beta>0$, and let $C_{\phi}: B^{\alpha} \rightarrow B^{\beta}$ be bounded.

(i) If $\phi$ is univalent and not a rotation, then $C_{\phi}$ is not an isometry.

(ii) If $\alpha \neq \beta$ and if $\exists M, r>0, r<1$ such that $\left|\phi^{\prime}(z)\right| \leq M$, whenever $|\phi(z)|>r$, then $C_{\phi}$ does not have a closed range.

Proof. (i) Let $\phi$ be univalent, not a rotation, and such that $C_{\phi}: \mathbb{B}^{\alpha} \rightarrow \mathbb{B}^{\beta}$ is an isometry. By Proposition 3.2, then $\phi(0)=0$ and $\sup _{z \in \mathbb{D}} \tau_{\phi, \alpha, \beta}(z)=1$. The function $f(z)=z$ is such that $\|f\|_{B^{\alpha}}=1$ and so

$$
1=\left\|C_{\phi} f\right\|_{\mathcal{B}^{\beta}}=\sup _{z \in \mathbb{D}} \tau_{\phi, \alpha, \beta}(z)\left(1-|\phi(z)|^{2}\right)^{\alpha}
$$

Since both parts of the last product are less or equal to 1 , there is a sequence $\left\{z_{n}\right\}$ in $\mathbb{D}$ such that $\tau_{\phi, \alpha, \beta}\left(z_{n}\right) \rightarrow 1$ and $\left|\phi\left(z_{n}\right)\right| \rightarrow 0$. Since $\phi$ is univalent, by the Köebe distortion theorem, it can not be that $\left|z_{n}\right| \rightarrow 1$. Hence, $\exists z_{0} \in \mathbb{D}$ such that $z_{n} \rightarrow z_{0}, \phi\left(z_{n}\right) \rightarrow \phi\left(z_{0}\right)=0$. Again, $\phi$ is univalent, and so $z_{0}=0$. But then $\tau_{\phi, \alpha, \beta}(0)=\left|\phi^{\prime}(0)\right|=1$ and by Schwarz lemma, $\phi$ has to be a rotation, which is a contradiction. Hence, $C_{\phi}$ is not an isometry.

(ii) Let $\alpha \neq \beta$ and let $\exists M, r>0, r<1$ such that $\left|\phi^{\prime}(z)\right| \leq M$, whenever $|\phi(z)|>r$. If $\|\phi\|_{\infty}<1$, then $C_{\phi}$ is compact and does not have a closed range. So, let $\zeta \in \partial \mathbb{D}$ be such that $|\phi(\zeta)|=1$.

If $\beta<\alpha$, the boundedness of the derivative as $|\phi(z)| \rightarrow 1$ implies that $\phi$ must have an angular derivative at $\zeta$, and by Proposition 3.5, $C_{\phi}$ is not bounded. 
If $\alpha<\beta$, then whenever $\phi\left(z_{n}\right) \rightarrow 1$,

$$
\tau_{\phi, \alpha, \beta}\left(z_{n}\right)=\frac{\left(1-\left|z_{n}\right|^{2}\right)^{\beta}\left|\phi^{\prime}\left(z_{n}\right)\right|}{\left(1-\left|\phi\left(z_{n}\right)\right|^{2}\right)^{\alpha}} \leq M\left(1-\left|z_{n}\right|^{2}\right)^{\beta-\alpha} \rightarrow 0 .
$$

Thus $C_{\phi}$ is compact and so it can not have a closed range.

Note that part (ii) of the previous theorem together with part (iii) of Theorem 3.3 show that, for example, if $\phi$ is a finite Blaschke product, then $C_{\phi}: B^{\alpha} \rightarrow B^{\beta}$ has a closed range only when $\alpha=\beta$.

We finish with the following two open questions.

Question 1. If the composition operator $C_{\phi}: \mathbb{B}^{\alpha} \rightarrow \mathbb{B}^{\beta}$ is bounded, is the necessary closedrange condition in part (ii) of Theorem 3.3 also sufficient for any $\alpha, \beta>0$ ? By Theorem $\mathrm{D}$, this is the case when $\alpha=\beta \geq 1$.

Question 2. Are there any closed-range composition operators $C_{\phi}: B^{\alpha} \rightarrow B^{\beta}$ when $\alpha$ and $\beta$ are such that either $0<\alpha<\beta<1$ or such that $1 \leq \beta<\alpha$ ? By Theorem 3.7 part (ii), if there is such a $C_{\phi}$, then $\phi$ will not be a finite Blaschke product.

\section{References}

[1] E. A. Nordgren, "Composition operators," Canadian Journal of Mathematics, vol. 20, pp. 442-449, 1968.

[2] M. J. Martín and D. Vukotić, "Isometries of some classical function spaces among the composition operators," in Recent Advances in Operator-Related Function Theory, vol. 393 of Contemp. Math., pp. 133138, Amer. Math. Soc., Providence, RI, USA, 2006.

[3] M. J. Martín and D. Vukotić, "Isometries of the Dirichlet space among the composition operators," Proceedings of the American Mathematical Society, vol. 134, no. 6, pp. 1701-1705, 2006.

[4] J. Laitila, "Isometric composition operators on BMOA," Mathematische Nachrichten, vol. 283, no. 11, pp. 1646-1653, 2010.

[5] J. R. Akeroyd and P. G. Ghatage, "Closed-range composition operators on $A^{2}$," Illinois Journal of Mathematics, vol. 52, no. 2, pp. 533-549, 2008.

[6] N. Zorboska, "Composition operators with closed range," Transactions of the American Mathematical Society, vol. 344, no. 2, pp. 791-801, 1994.

[7] C. C. Cowen and B. D. MacCluer, Composition Operators on Spaces of Analytic Functions, Studies in Advanced Mathematics, CRC Press, Boca Raton, Fla, USA, 1995.

[8] J. H. Shapiro, Composition Operators and Classical Function Theory, Universitext: Tracts in Mathematics, Springer, New York, NY, USA, 1993.

[9] K. H. Zhu, Operator Theory in Function Spaces, vol. 139 of Monographs and Textbooks in Pure and Applied Mathematics, Marcel Dekker, New York, NY, USA, 1990.

[10] K. H. Zhu, "Bloch type spaces of analytic functions," The Rocky Mountain Journal of Mathematics, vol. 23, no. 3, pp. 1143-1177, 1993.

[11] K. M. Madigan, "Composition operators on analytic Lipschitz spaces," Proceedings of the American Mathematical Society, vol. 119, no. 2, pp. 465-473, 1993.

[12] K. Madigan and A. Matheson, "Compact composition operators on the Bloch space," Transactions of the American Mathematical Society, vol. 347, no. 7, pp. 2679-2687, 1995.

[13] R. C. Roan, "Composition operators on a space of Lipschitz functions," The Rocky Mountain Journal of Mathematics, vol. 10, no. 2, pp. 371-379, 1980.

[14] J. H. Shapiro, "Compact composition operators on spaces of boundary-regular holomorphic functions," Proceedings of the American Mathematical Society, vol. 100, no. 1, pp. 49-57, 1987.

[15] J. Xiao, "Composition operators associated with Bloch-type spaces," Complex Variables. Theory and Application, vol. 46, no. 2, pp. 109-121, 2001. 
[16] M. J. Martín and D. Vukotić, "Isometries of the Bloch space among the composition operators," Bulletin of the London Mathematical Society, vol. 39, no. 1, pp. 151-155, 2007.

[17] J. A. Cima and W. R. Wogen, "On isometries of the Bloch space," Illinois Journal of Mathematics, vol. 24, no. 2, pp. 313-316, 1980.

[18] J. M. Cohen and F. Colonna, "Preimages of one-point sets of Bloch and normal functions," Mediterranean Journal of Mathematics, vol. 3, no. 3-4, pp. 513-532, 2006.

[19] F. Colonna, "Characterisation of the isometric composition operators on the Bloch space," Bulletin of the Australian Mathematical Society, vol. 72, no. 2, pp. 283-290, 2005.

[20] N. Zorboska, "Isometric composition operators on the Bloch-type spaces," Comptes Rendus Mathématiques de l'Académie des Sciences. La Société Royale du Canada, vol. 29, no. 3, pp. 91-96, 2007.

[21] H. Chen, "Boundedness from below of composition operators on the Bloch spaces," Science in China. Series A, vol. 46, no. 6, pp. 838-846, 2003.

[22] P. Ghatage, J. Yan, and D. Zheng, "Composition operators with closed range on the Bloch space," Proceedings of the American Mathematical Society, vol. 129, no. 7, pp. 2039-2044, 2001.

[23] P. Ghatage, D. Zheng, and N. Zorboska, "Sampling sets and closed range composition operators on the Bloch space," Proceedings of the American Mathematical Society, vol. 133, no. 5, pp. 1371-1377, 2005.

[24] H. Chen and P. Gauthier, "Boundedness from below of composition operators on $\alpha$-Bloch spaces," Canadian Mathematical Bulletin, vol. 51, no. 2, pp. 195-204, 2008.

[25] N. Zorboska, "Univalently induced closed range composition operators on the Bloch-type spaces," Canadian Mathematical Bulletin, vol. 54, no. 2, 2011. 


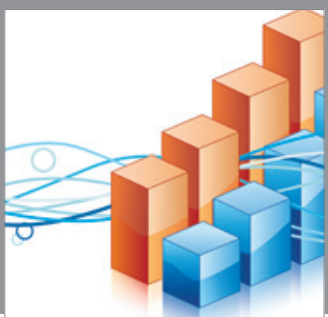

Advances in

Operations Research

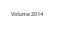

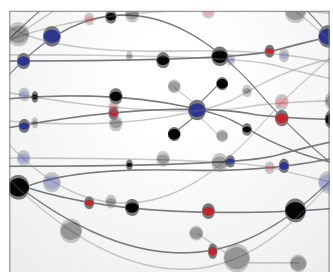

\section{The Scientific} World Journal
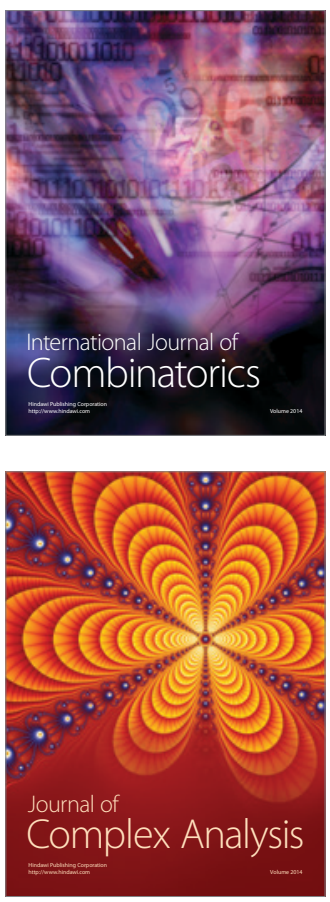

International Journal of

Mathematics and

Mathematical

Sciences
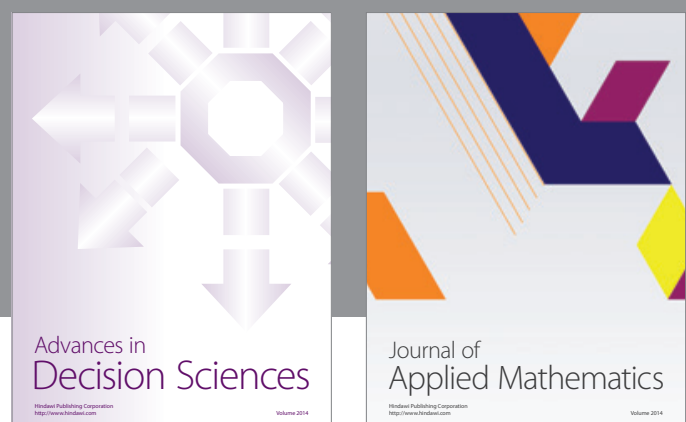

Journal of

Applied Mathematics
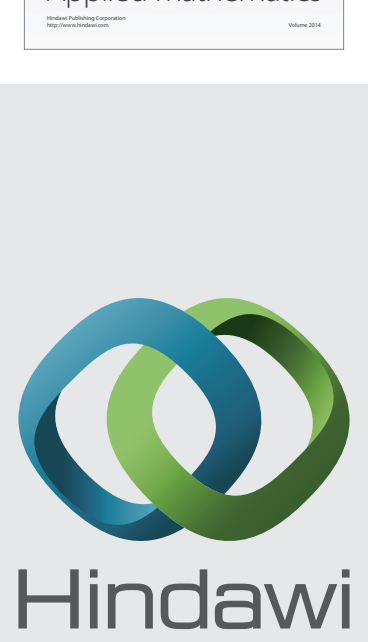

Submit your manuscripts at http://www.hindawi.com
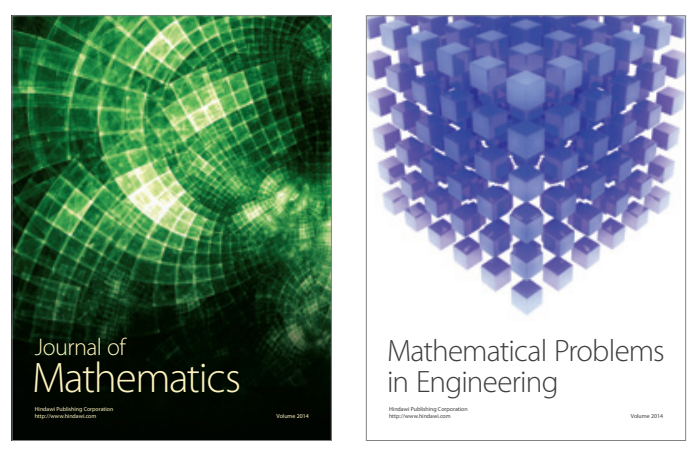

Mathematical Problems in Engineering
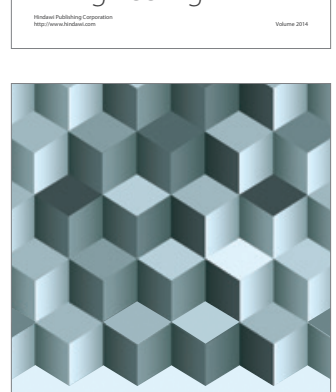

Journal of

Function Spaces
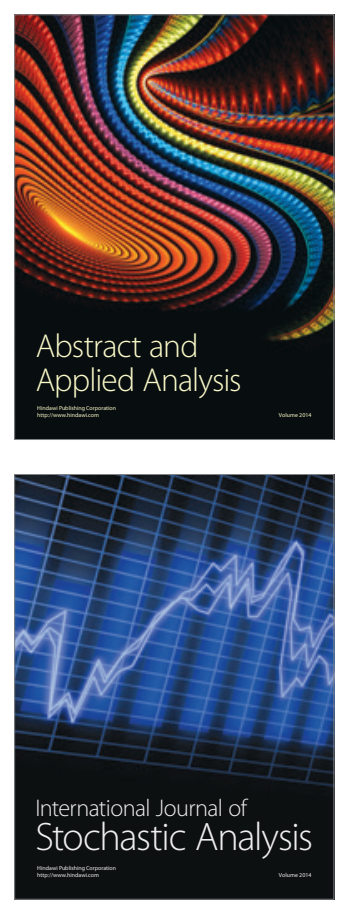

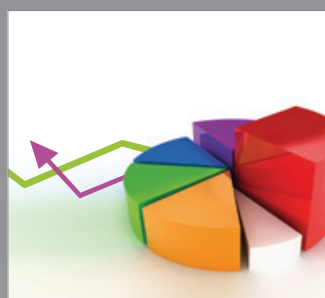

ournal of

Probability and Statistics

Promensencen
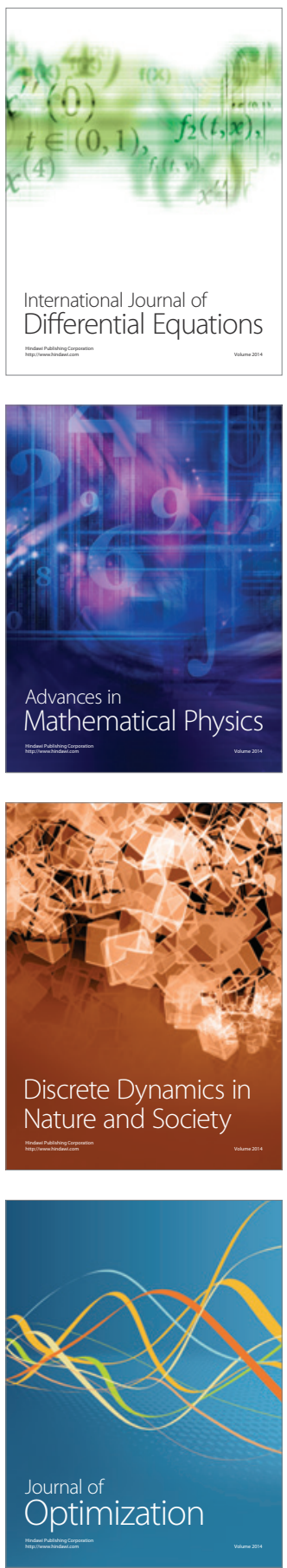